\title{
The phenotypic spectrum of Schaaf-Yang syndrome: 18 new affected individuals from 14 families
}

\author{
Michael D. Fountain ${ }^{1,2,3}$, Emmelien Aten, MD, PhD ${ }^{4}$, Megan T. Cho, ScM, CGC ${ }^{5}$, \\ Jane Juusola, PhD5, Magdalena A. Walkiewicz, PhD², Joseph W. Ray, MD ${ }^{6,7}$, Fan Xia, PhD², \\ Yaping Yang, PhD², Brett H. Graham, MD, PhD ${ }^{2,8}$, Carlos A. Bacino, MD2,8, Lorraine Potocki, MD ${ }^{2,8}$, \\ Arie van Haeringen, MD, Claudia A.L. Ruivenkamp, PhD ${ }^{4}$, Pedro Mancias, MD , Hope Northrup, MD, \\ Mary K. Kukolich, MD ${ }^{9}$, Marjan M. Weiss, MD ${ }^{10}$, Conny M.A. van Ravenswaaij-Arts, MD, PhD ${ }^{11}$, \\ Inge B. Mathijssen, $\mathrm{MD}^{12}$, Sebastien Levesque, MD, PhD ${ }^{13}$, Naomi Meeks, MD ${ }^{14}$, Jill A. Rosenfeld, MS, CGC ${ }^{2}$, \\ Danielle Lemke, MS, CGC ${ }^{14}$, Ada Hamosh, MD, MPH${ }^{15}$, Suzanne K. Lewis, MD ${ }^{16}$, Simone Race, MS, $\mathrm{CGC}^{16}$, \\ Laura L. Stewart, MD ${ }^{17}$, Beverly Hay, MD ${ }^{18}$, Andrea M. Lewis, MS, CGC ${ }^{2,8}$, Rita L. Guerreiro, PhD ${ }^{19}$, \\ Jose T. Bras ${ }^{19}$, Marcia P. Martins, MD ${ }^{20}$, Gerarda Derksen-Lubsen, MD, PhD ${ }^{21}$, Els Peeters, MD ${ }^{22}$, \\ Connie Stumpel, MD, PhD²3, Sander Stegmann, PhD ${ }^{23}$, Levinus A. Bok, MD²4, \\ Gijs W.E. Santen, MD, $\mathrm{PhD}^{4}$ and Christian P. Schaaf, MD, PhD ${ }^{1,2,3}$
}

\begin{abstract}
Purpose: Truncating mutations in the maternally imprinted, paternally expressed gene MAGEL2, which is located in the Prader-Willi critical region 15q11-13, have recently been reported to cause Schaaf-Yang syndrome, a Prader-Willi-like disease that manifests as developmental delay/intellectual disability, hypotonia, feeding difficulties, and autism spectrum disorder. The causality of the reported variants in the context of the patients' phenotypes was questioned, as MAGEL2 whole-gene deletions seem to cause little or no clinical phenotype.

Methods: Here we report a total of 18 newly identified individuals with Schaaf-Yang syndrome from 14 families, including 1 family with 3 individuals found to be affected with a truncating variant of MAGEL2, 11 individuals who are clinically affected but were not tested molecularly, and a presymptomatic fetal sibling carrying the pathogenic MAGEL2 variant.
\end{abstract}

\section{INTRODUCTION}

Truncating pathogenic variants in the maternally imprinted, paternally expressed MAGEL2 gene were initially reported as the cause of developmental delay (DD)/intellectual disability
Results: All cases harbor truncating mutations of MAGEL2, and nucleotides c.1990-1996 arise as a mutational hotspot, with 10 individuals and 1 fetus harboring a c.1996dupC (p.Q666fs) mutation and 2 fetuses harboring a c.1996delC (p.Q666fs) mutation. The phenotypic spectrum of Schaaf-Yang syndrome ranges from fetal akinesia to neurobehavioral disease and contractures of the small finger joints.

Conclusion: This study provides strong evidence for the pathogenicity of truncating mutations of the paternal allele of MAGEL2, refines the associated clinical phenotypes, and highlights implications for genetic counseling for affected families.

Genet Med advance online publication 19 May 2016

Key Words: MAGEL2; neurodevelopment; Prader-Willi syndrome; Schaaf-Yang syndrome

(ID), hypotonia, feeding difficulties, and autism spectrum disorder (ASD) in four reported individuals. ${ }^{1}$ Because of phenotypic overlap with Prader-Willi syndrome (PWS (OMIM 176270)), and because MAGEL2 is one of the imprinted,

\footnotetext{
The first two authors are co-first authors.

${ }^{1}$ Interdepartmental Program in Translational Biology and Molecular Medicine, Baylor College of Medicine, Houston, Texas, USA; ${ }^{2}$ Department of Molecular and Human Genetics, Baylor College of Medicine, Houston, Texas, USA; ${ }^{3}$ Jan and Dan Duncan Neurological Research Institute, Texas Children's Hospital, Houston Texas, USA; ${ }^{4}$ Department of Clinical Genetics, Leiden University Medical Center, Leiden, The Netherlands; ${ }^{5}$ GeneDX, Gaithersburg, Maryland, USA; ${ }^{6}$ Division of Medical Genetics, University of Texas Medical Branch, Galveston, Texas, USA; ${ }^{7}$ Department of Pediatrics, The University of Texas Medical School at Houston, Houston, Texas, USA; ${ }^{8}$ Texas Children's Hospital, Houston, Texas, USA; ${ }^{9}$ Genetic Services, Cook Children's Health Care System, Fort Worth, Texas, USA; ${ }^{10}$ Clinical Genetics, VU University Medical Center, Amsterdam, The Netherlands; ${ }^{11}$ Department of Genetics, University Medical Center Groningen, University of Groningen, Groningen, The Netherlands; ${ }^{12}$ Department of Clinical Genetics, Academic Medical Center, Amsterdam, The Netherlands; ${ }^{13}$ Département de pédiatrie, Centre Hospitalier Universitaire de Sherbrooke, Sherbrooke, Quebec, Canada; ${ }^{14}$ Children's Hospital Colorado, Aurora, Colorado, USA; ${ }^{15}$ McKusick-Nathans Institute of Genetic Medicine, Johns Hopkins University School of Medicine, Baltimore, Maryland, USA; ${ }^{16}$ Department of Medical Genetics, BC Children's and Women's Health Center of British Columbia, The University of British Columbia, Vancouver, British Columbia, Canada; ${ }^{17}$ Department of Pediatrics, BC Children's and Women's Health Center of British Columbia, The University of British Columbia, Vancouver, British Columbia, Canada; ${ }^{18}$ Division of Genetics, UMass Memorial Children's Medical Center, University of Massachusetts Medical School, Worcester, Massachusetts, USA; ${ }^{19}$ Department of Molecular Neuroscience, University College London Institute of Neurology, London, UK; ${ }^{20}$ Centro Hospitalar de Trás-os-Montes e Alto Douro, Unidade Hospital de Vila Real, Vila Real, Portugal; ${ }^{21}$ Department of Pediatrics, Juliana Children's Hospital-Haga Teaching Hospital, The Hague, The Netherlands; ${ }^{22}$ Department of Child Neurology, Juliana Children's Hospital-Haga Teaching Hospital, The Hague, The Netherlands; ${ }^{23}$ Department of Clinical Genetics and School for Oncology and Developmental Biology, Maastricht UMC+, Maastricht, The Netherlands; ${ }^{24}$ Department of Pediatrics, Máxima Medical Center, Veldhoven, The Netherlands. Correspondence: Christian P. Schaaf (schaaf@bcm.edu) Or Gijs W.E. Santen (G.W.E.Santen@lumc.nl)
} 
protein-coding genes in the Prader-Willi domain on chromosome 15q11-q13, the condition was initially considered a Prader-Willi-like syndrome.

PWS is characterized by neonatal hypotonia, failure to thrive, hyperphagia and excessive weight gain in childhood, DD/ID, hypogonadism, short stature, and a unique behavioral profile. ${ }^{2}$ While the four previously reported individuals with truncating MAGEL2 mutations exhibited marked phenotypic overlap with PWS, including neonatal hypotonia, poor suck requiring special feeding techniques, DD/ID, hypogonadism, and excessive weight gain, they also manifested phenotypes that seemed to be distinct from PWS. These phenotypes included ASD, present in all four individuals, and contractures of the small finger joints, present in three. Other characteristics of PWS, such as the typical hyperphagia and subsequent obesity, were either absent or only mildly expressed. To highlight the phenotypic differences between PWS and the clinical condition caused by truncating MAGEL2 mutations, the latter was renamed Schaaf-Yang syndrome (SHFYNG (OMIM 615547)).

To date, a total of 10 individuals with pathogenic variants in MAGEL2 have been reported. ${ }^{1,3,4}$ Six were diagnosed during childhood or adolescence with clinical features of SHFYNG, and another four children from two unrelated families manifested severe arthrogryposis.

Importantly, the pathogenicity of truncating mutations of the paternal allele of MAGEL2 has been questioned because of the small number of cases reported and because whole-gene deletions of the paternal copy of MAGEL2 seem to have no phenotype or very mild phenotypes. ${ }^{5,6}$

Here we report 18 newly identified individuals with truncating mutations in MAGEL2 (Figure 1a). Among these are three families with multiple affected individuals, including one family with 3 molecularly confirmed individuals, 11 who are clinically affected but have not been tested molecularly, a fetus with a prenatal molecular diagnosis, and an inheritance pattern consistent with SHFYNG (Figure 1b). Our study provides strong evidence for the pathogenicity of MAGEL2 mutations. It illustrates the broad phenotypic spectrum of SHFYNG, which ranges from fetal akinesia and arthrogryposis multiplex congenita to mild ID and contractures of the small finger joints. Postnatal complications, including premature lethality, are discussed.

\section{MATERIALS AND METHODS}

Individuals with truncating MAGEL2 variants were enrolled based on genotype. Of the 14 probands reported in this study, 12 were identified by clinical whole-exome sequencing and 2 by targeted, clinical Sanger sequencing of the MAGEL2 gene. Five probands were enrolled through the Baylor Miraca Genetics Laboratories and four probands were enrolled through the GeneDx laboratory, of whom one was enrolled through GeneMatcher. One family was identified at the Leiden University Medical Center. Cases identified by whole-exome sequencing were subsequently confirmed by Sanger sequencing. Additional affected family members were identified by targeted Sanger sequencing of the known familial mutations. Upon identification of a probable pathogenic variant in MAGEL2, genetic counselors from the respective laboratories and institutions contacted the referring providers, informing them of a research interest in Dr Schaaf's laboratory. Four families contacted Dr Schaaf directly because of a new diagnosis of SHFYNG made by molecular genetic testing. An initial phenotypic assessment was obtained of all newly identified and molecularly confirmed individuals harboring pathogenic variants in MAGEL2 (patients 1-18 presented in Supplementary Tables S1-S3 online). Upon consent to share clinical information, all were contacted for a detailed review of the medical and family histories, and to ascertain developmental milestones and physical features. Medical records were reviewed. Written informed consent was obtained for the use of the medical history, the genetic testing report, and a photograph (if applicable), as approved by the institutional review board at Baylor College of Medicine.

\section{RESULTS}

Combining the phenotypic characteristics of the novel 18 individuals with pathogenic variants in MAGEL2 reported herein with those previously identified by Schaaf et al., ${ }^{1}$ Soden et al., ${ }^{3}$ and Mejlachowicz et al., ${ }^{4}$ we are able to delineate the physical, cognitive, and behavioral spectrum of phenotypes associated with SHFYNG (Table 1). DD, ID, and hypotonia represent the most common phenotypes and are present among all individuals for whom this information has been available (Table 1).

On average, affected children sat independently at 19 months (range: 8-36 months), walked at 39 months (range: 24-60 months; patients 16 ( 15 years old) and 18 (20 years old) cannot walk), and spoke their first word at 56 months (range: 12-144 months; patients 10 (10 years old), 16 (15 years old), and 18 (20 years old) cannot speak). The level of ID ranged from mild to severe. Of the newly identified cases with both phenotypic information and a confirmed molecular diagnosis, seven were evaluated for ASD. Of these seven individuals, four received a formal diagnosis of ASD. An additional six subjects were described as manifesting symptoms of ASD (denoted with an asterisk in Table 1); however, no formal evaluation was performed, and further assessment is necessary. An analysis of patient behavior identified a spectrum of abnormalities, including impulsive, compulsive, stubborn, and manipulative behaviors (seen in 9 of 11 cases for which this information was available). Parents reported habitual skin picking or automutilation of varying severity in 7 of 12 molecularly diagnosed individuals.

Neonatal hypotonia was identified as an early, initial physical phenotype displayed by patients with pathogenic variants in MAGEL2. ${ }^{1,3}$ Among the molecularly confirmed individuals in this study, 14 of 15 manifested neonatal hypotonia. Fifteen of 16 displayed feeding difficulties requiring special feeding techniques. Joint contractures were present in 15 of 18 of these patients. The severity of contractures ranged from affecting 
a

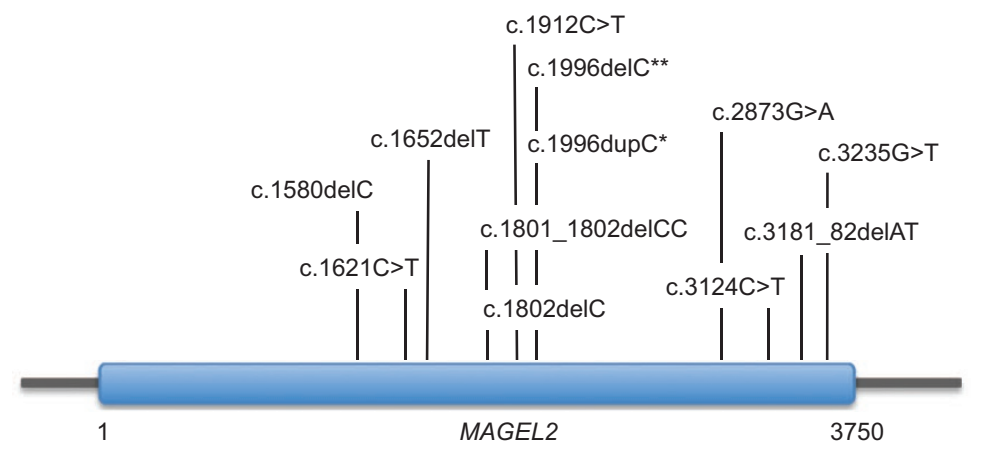

b

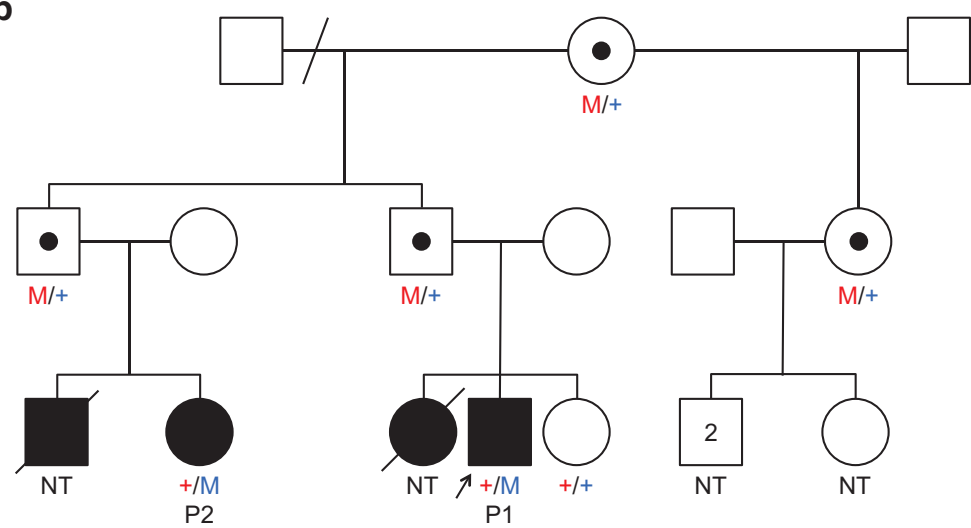

Familial mutation: c.1996dupC

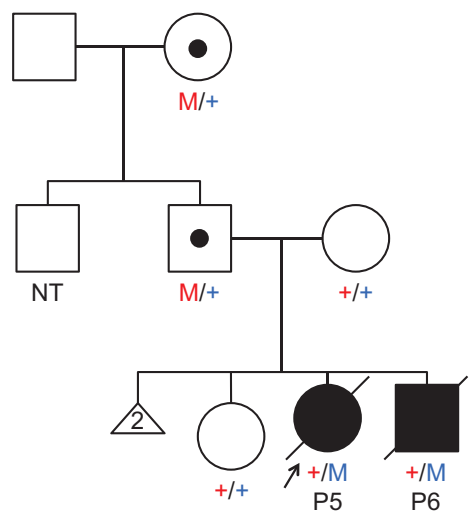

Familial mutation: c.1996delC

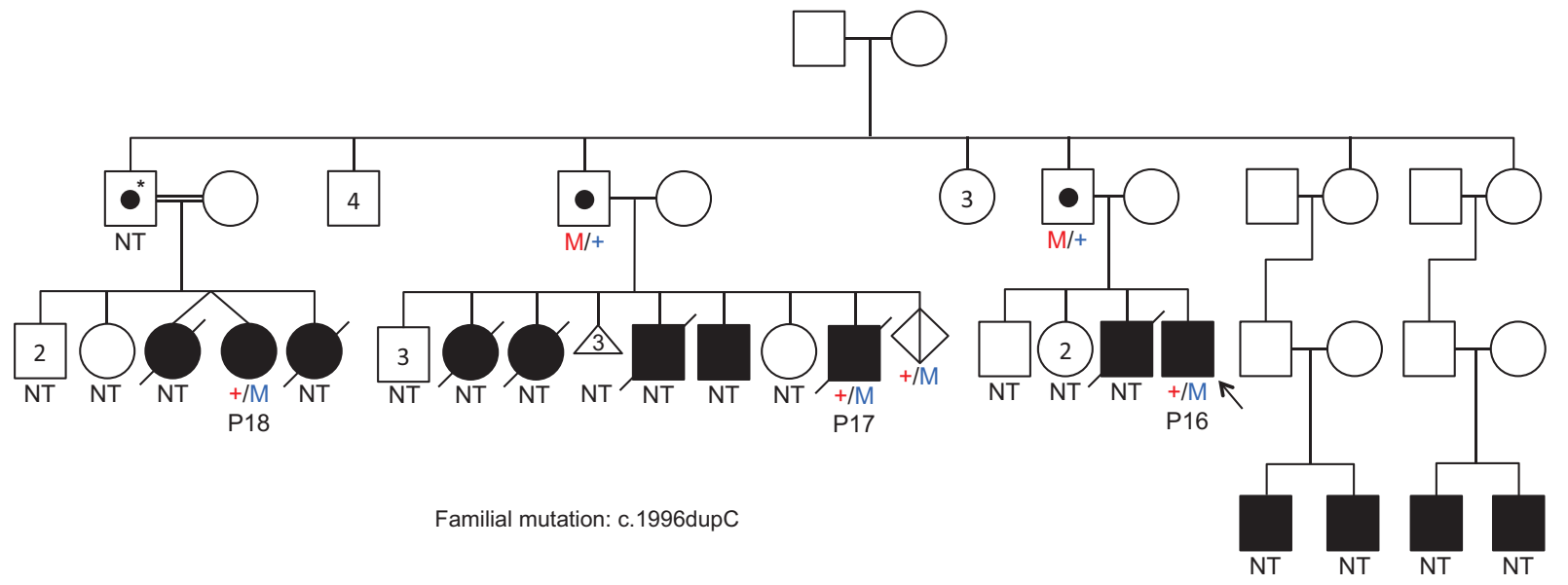

Figure 1 Newly identified truncating mutations of MAGEL2 in Schaaf-Yang syndrome. (a) Truncating MAGEL2 mutations reported in this article are indicated by their positions in the coding sequence of the gene. *Individuals with a c.1996dupC mutation $(n=11) . * *$ Individuals with a c. 1996delC mutation $(n=2)$. (b) Pedigrees of familial cases. Pedigree 1, family of patients 1 and 2; pedigree 2, family of patients 5 and 6; pedigree 3, family of patients 16-18. Black squares and circles indicate individuals who are/were clinically affected. Black dots indicate carrier individuals who harbor the familial MAGEL2 mutation on their maternal allele. Maternal alleles are indicated in red, paternal alleles in blue. +, reference sequence for MAGEL2; M, familial MAGEL2 mutation; NT, not tested; P, patient. *Obligate carrier.

only the interphalangeal joints to fetal akinesia with severe arthrogryposis (present in patients 5 and 6, discussed below). Additional abnormalities of the hands included tapering fingers, clinodactyly, camptodactyly, brachydactyly, and adducted thumbs (surgically corrected in patient 3 ) (Figure 2). A further assessment of skeletal features showed that 12 of 14 patients had small hands, 8 of 15 had small feet, and 10 of 16 cases had short stature. Further, 7 of 14 patients were diagnosed with scoliosis or kyphosis. Sleep abnormalities were common, with 9 of 13 patients diagnosed with sleep apnea. Hypogonadism in the form of cryptorchidism and/or micropenis was present in 8 of 11 male patients and represents one of the earliest recognized physical features immediately after birth. In addition, patient 18 (Supplementary Table $\mathbf{S 3}$ online) is a 20 -year-old female diagnosed with hypogonadotropic hypogonadism. Physical examination showed dysmorphic labial development. She had 
Table 1 Summary of published and novel patients with deleterious variants in MAGEL2

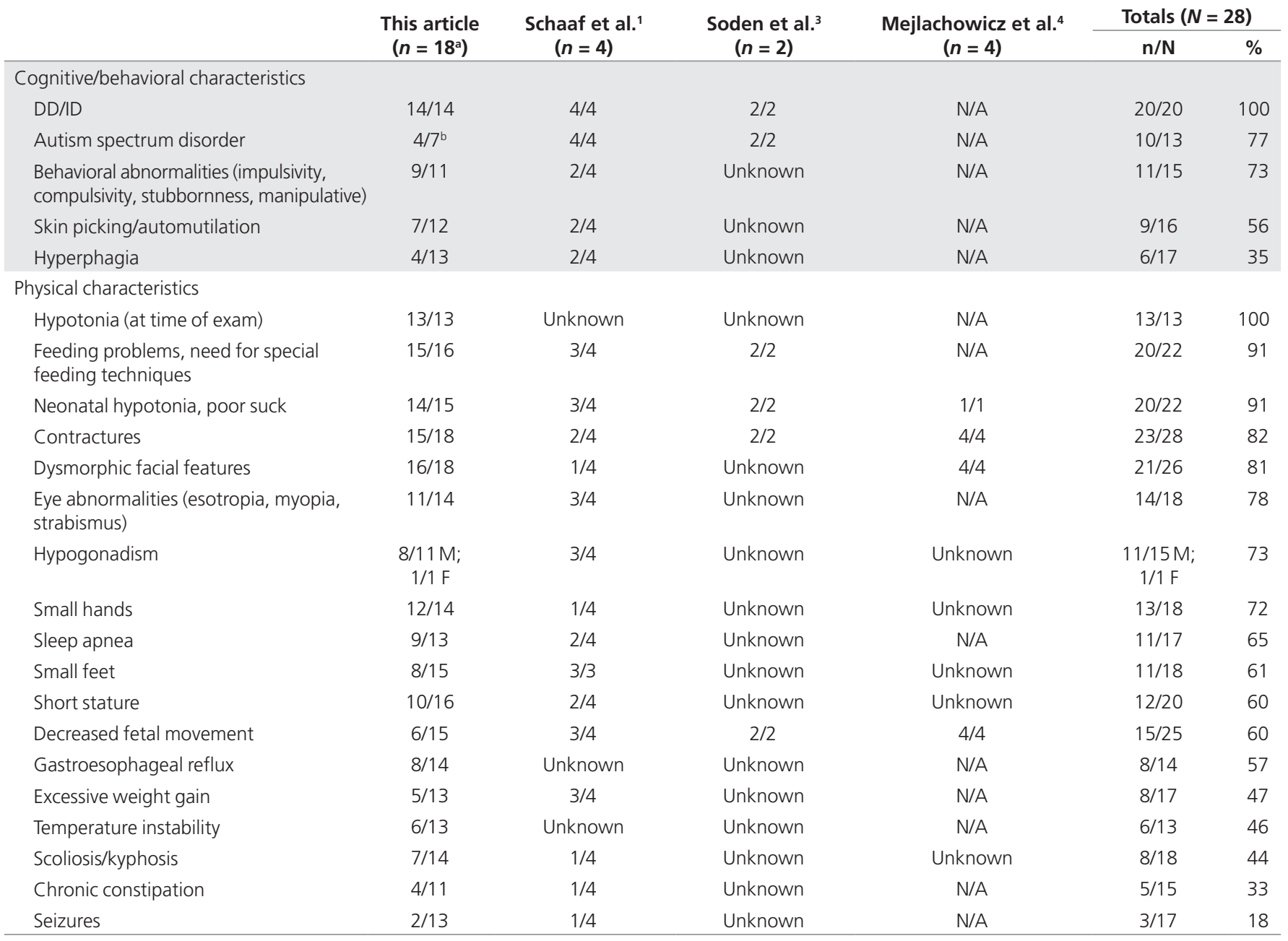

anly individuals with a molecularly confirmed MAGEL2 mutation and a detailed medical history are included in this table. ${ }^{\mathrm{b}} \mathrm{Seven}$ subjects received formal testing; six more patients express symptoms of autism spectrum disorder.

DD/ID, developmental delay/intellectual disability; F, female; M, male; N/A, not applicable.

spontaneous menarche but no spontaneous development of secondary sex characteristics. This was stimulated with estrogen supplementation at 18 years, and she now uses oral contraceptives. Eleven of 14 patients manifested eye abnormalities in the form of strabismus, esotropia, or myopia.

Of 18 patients with a molecularly confirmed postnatal diagnosis, 16 are described to have a spectrum of varying facial dysmorphisms, including malformations of the philtrum, ear position, nasal structure, frontal bossing, and palpebral fissure length. As well, prognathia with a squared-off chin appears to be present in the majority of older children. As evidenced in Figure 3, there is an appreciable variability in the degree of these facial dysmorphisms, as well as bushy eyebrows and short noses.

\section{Familial cases}

Patients 1 and 2 are paternal cousins who both carry a deleterious c.1996dupC (p.Q666fs) variant in the MAGEL2 gene (Figure 1b, pedigree on the upper left; Supplementary Table S4 online), detected by clinical whole-exome sequencing in the propositus (patient 1). Patient 1 is a 15 -year-old male presenting characteristic phenotypes of SHFYNG, specifically neonatal hypotonia, feeding difficulties requiring assisted feeding techniques, contractures, hypotonia at the time of the exam, and hypogonadism, among others (Figure 3a). In addition, he manifested cognitive and behavioral phenotypes typical of SHFYNG, namely DD/ID, ASD, and a behavioral profile including impulsivity, compulsivity, stubbornness, and manipulative behaviors. He did not have any indication of hyperphagia (Supplementary Table S1 online). Patient 2 is a 6-year-old female who shares several physical characteristics with her male cousin. She manifested neonatal hypotonia, feeding difficulties requiring assistance, contractures, and hypotonia at the time of the exam, among other phenotypes (Figures 2a and $3 \mathbf{b}$ ). As well, she manifested DD/ID and ASD, but no hyperphagia (Supplementary Table S1 online). 


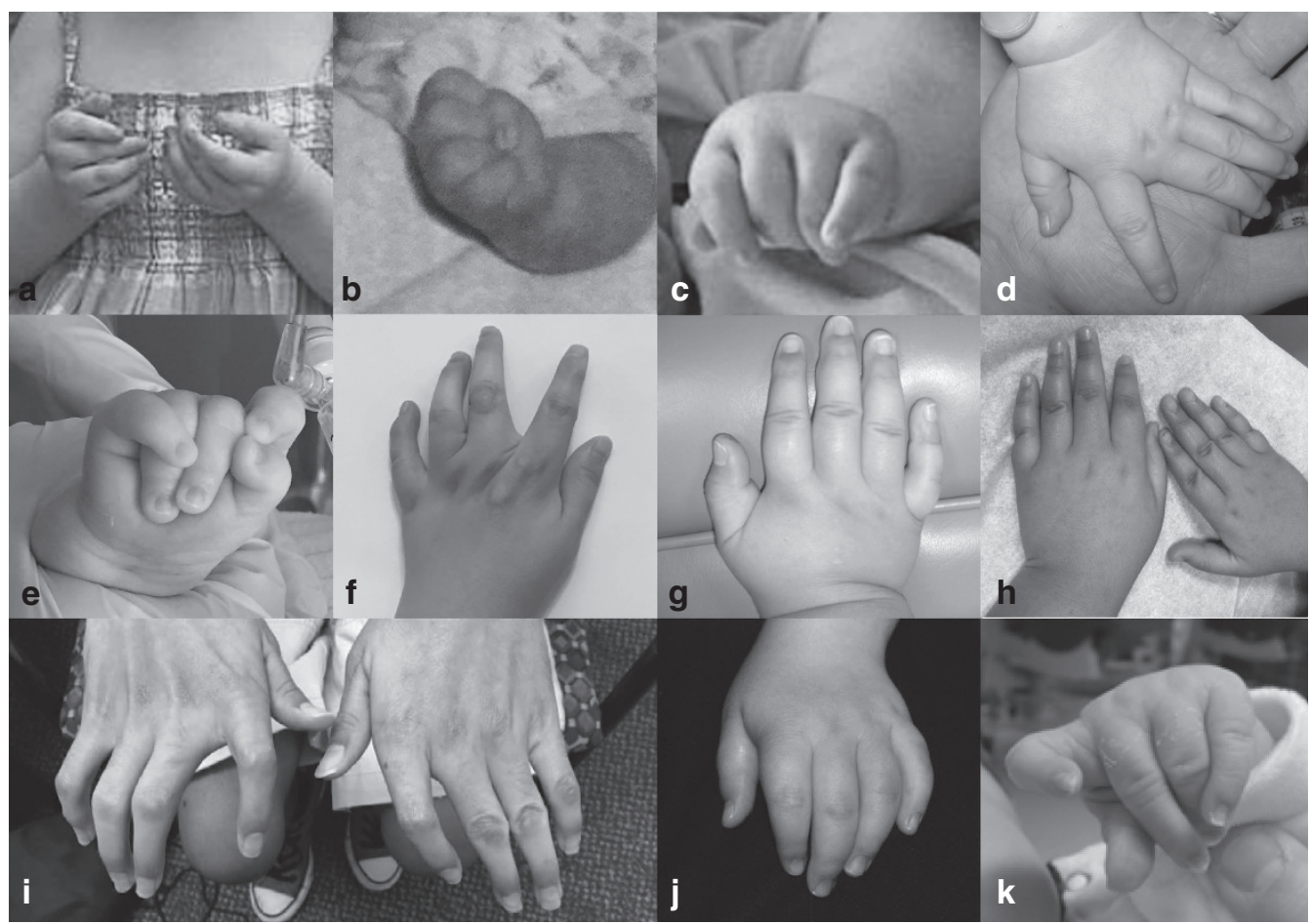

Figure 2 Hand phenotypes in patients with Schaaf-Yang syndrome. Affected patients manifest a spectrum of phenotypes, including contractures of the interphalangeal joints, camptodactyly, tapering of the fingers, brachydactyly, clinodactyly, and adducted thumbs: (a) patient 2; (b) patient 3; (c) patient 4; (d) patient 7; (e) patient 9; (f) patient 10; (g) patient 11; (h) patient 13; (i) patient 14; (j) patient 15; (k) patient 17.

Using methylation-sensitive digestion of the genomic DNA followed by polymerase chain reaction amplification and Sanger sequencing, as previously described, ${ }^{1}$ we confirmed that the c.1996dupC variant was present on the paternal MAGEL2 allele of patient 1 . Subsequent analysis revealed that his father and paternal grandmother were both carriers of the c.1996dupC (p.Q666fs) change and that the mutation resided on their maternal chromosomes 15. This finding is consistent with their clinically unaffected status, based on the molecular mechanism of this disorder, caused by mutations of a paternally expressed, maternally imprinted gene. We then established that the proband's paternal uncle and half-aunt were also carriers of the c.1996dupC (p.Q666fs) mutation, which they had inherited from their mother. Molecular analysis of the patient's affected female cousin (patient 2; Supplementary Table S1 online) showed that she had inherited the c.1996dupC (p.Q666fs) mutation from her clinically unaffected carrier father.

Patients 5 and 6 are fetal cases (from two separate pregnancies) affected with fetal akinesia and severe arthrogryposis (Figure $1 \mathrm{~b}$, pedigree on the upper right). Analysis via wholeexome sequencing revealed that both fetuses harbored a deleterious variant, c.1996delC (p.Q666fs), in the MAGEL2 gene. Patient 5 was a female fetus presenting with fetal akinesia, noted on prenatal ultrasound examination (Supplementary Table S1 online; Supplementary Figure S1a,b online). Upon consultation, the family elected to terminate the pregnancy at 21 weeks and 4 days of gestation. A postmortem examination identified contractures, overlapping digits, and rocker-bottom feet, as well as retro-micrognathia and gnatho-palatoschisis. Patient 6 , a male sibling of patient 5 , also presented with fetal akinesia (Supplementary Table S1 online; Supplementary Figure S1 c,d online), and the family elected to terminate the pregnancy at 14 weeks and 6 days of gestation. A postmortem examination identified a similar phenotypic expression with contractures, overlapping digits, and retro-micrognathia. Moreover, patient 6 manifested multiple pterygia and bilateral congenital talipes equinovarus. The c.1996delC (p.Q666fs) variant identified in patients 5 and 6 was found to be inherited from the unaffected father and the unaffected paternal grandmother. Moreover, the variant was found not to be present in the healthy sibling to these fetuses.

Patients 16, 17, and 18 are paternal cousins (Figure 1b, lower pedigree). Whole-exome sequencing identified the c.1996dupC (p.Q666fs) mutation in the proband (patient 16) and patient 18. Molecular analysis confirmed the mutation in patient 17 , the proband's father, and one paternal uncle. Sanger sequencing identified the mutation in a chorionic villus sample of a fetal sibling of patient 17 (Figure 1b, lower pedigree), who shows a clenched fist on ultrasound examination at 29 weeks and 6 days of gestation. Patients 16, 17, and 18 manifested phenotypes consistent with SHFYNG, most notably, neonatal hypotonia, feeding difficulties requiring assisted feeding techniques, $\mathrm{DD} / \mathrm{ID}$, dysmorphic facial features, and contractures (Supplementary Table S3 online; Figures $2 \mathrm{k}$ and 31 ). Patient 17, a deceased 9-month-old male, died with an unknown cause of death, though apnea was suspected. 


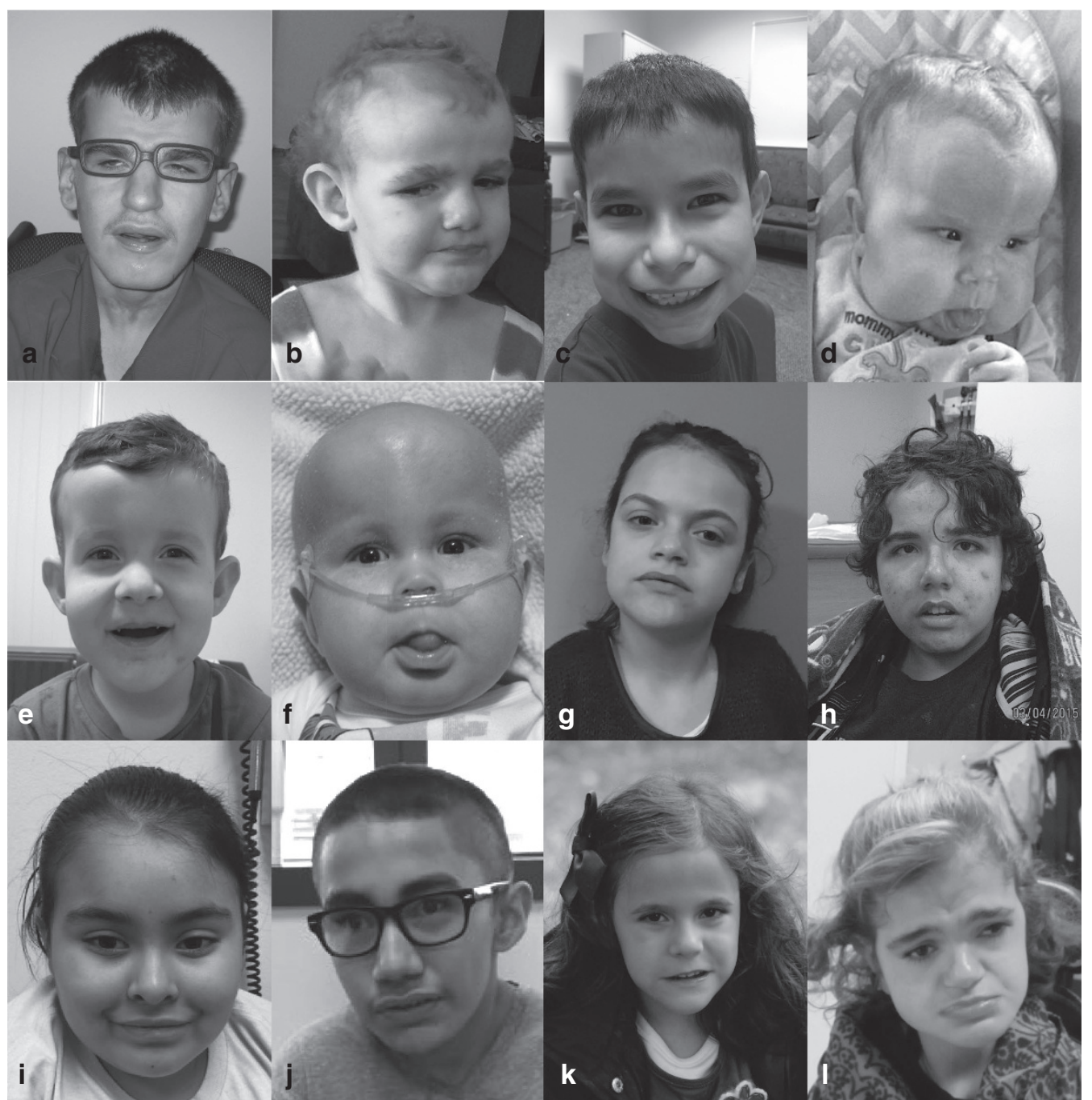

Figure 3 Facial phenotype of individuals with Schaaf-Yang syndrome. While several individuals manifest dysmorphic facial features, such as short noses, bushy eyebrows, and prognathism, the consistency of facial characteristics across individuals is limited: (a) patient 1; (b) patient 2; (c) patient 3; (d) patient 4; (e) patient 7; (f) patient 9; (g) patient 10; (h) patient 11; (i) patient 13; (j) patient 14; (k) patient 15; (I) patient 18.

To appreciate the prevalence of SHFYNG among samples submitted for clinical whole-exome sequencing, we analyzed the data available from the Baylor Miraca Genetics and the GeneDx laboratories. As of February 2016, the two laboratories have analyzed a combined total of 15,360 proband exomes. Of these 15,360 individuals, 13 were identified to have pathogenic truncating variants in MAGEL2. When filtering for those manifesting DD and/or ID and hypotonia, 7 of 3,820 individuals were identified as having pathogenic MAGEL2 variants. When filtering for male individuals with DD and/or ID and hypogonadism, 4 of 162 individuals were identified as positive for MAGEL2 truncating variants. This analysis is limited by the phenotypes listed at the time of sample submission.

\section{DISCUSSION}

In this report we present 18 individuals with phenotypic characteristics of SHFYNG, all of whom have molecularly confirmed truncating mutations in MAGEL2. The consistency of clinical features among affected individuals, and the segregation of clinical phenotypes with the presence of the respective MAGEL2 mutations on the paternal allele in three families, provides evidence of the pathogenicity of these mutations. Furthermore, the consistency of clinical features suggests a clinically recognizable syndrome characterized by neonatal hypotonia, feeding difficulties, contractures, and developmental delay.

Previously reported patients with deletions encompassing MAGEL2, but not the SNORD116@ cluster on chromosome $15 \mathrm{q}$, seem to have milder phenotypes than those individuals with truncating MAGEL2 mutations. ${ }^{5,6}$ Buiting et al. ${ }^{6}$ described a patient with deletion of MAGEL2 who presented with transient hypotonia and feeding difficulties during infancy. He had kyphosis and manifested a slight delay in fine and gross motor skills. The concept that a deletion of the entire gene could have milder effects than a truncating mutation is intriguing. Because MAGEL2 is a one-exon gene, one could speculate that truncating mutations may result in a truncated protein, which then 
could have a dominant-negative effect. Alternatively, the deletion of the entire paternal copy of this imprinted gene, including its promoter, could lead to leaky expression of the maternal copy of the gene. This has been suggested based on studies of Magel $2 m+/ p-$ mice. ${ }^{7}$ Indeed, further investigation of individuals with MAGEL2 deletions is necessary and may broaden the phenotypic spectrum associated with MAGEL2 mutations.

To further delineate the clinical phenotype associated with MAGEL2 truncating variants, we report prominent early phenotypes of fetal akinesia, neonatal hypotonia, feeding difficulties requiring assisted feeding techniques, and DD/ID. Sleep apnea, gastroesophageal reflux, and decreased fetal movement are frequently reported in SHFYNG. These symptoms may be observed because of the muscular dysfunction associated with the early hypotonia. Importantly, 23 of 28 postnatal individuals with molecularly confirmed MAGEL2 mutations reported to date manifest contractures of their interphalangeal joints, a phenotype rarely reported among individuals with $\mathrm{PWS},{ }^{8-10}$ providing further evidence that the prevalence of certain phenotypes may be markedly different between SHFYNG and PWS.

Although the infantile phenotype of SHFYNG is profoundly similar to that of PWS, the majority of patients with point mutations in MAGEL2 seem not to go through the later nutritional stages of PWS. ${ }^{11}$ In particular, most individuals seem not to progress to phase III, which is characterized by hyperphagia. Further, only 8 of 17 individuals with a molecularly confirmed diagnosis had excessive weight gain, which is typically associated with PWS. Interestingly, patient 1 described here and the siblings recently described by Soden et al. ${ }^{3}$ (CMH382 and CMH382) had unusual body habitus with increased abdominal girth. Body composition and patterns of adiposity have not been studied in individuals with MAGEL2 mutations, but this should be considered, taking into account the murine phenotype of Magel2 loss of function. The mice are not significantly overweight but do have increased adiposity with altered metabolism in adulthood. ${ }^{12}$ Strikingly, 10 of 13 patients tested to date carry a diagnosis of ASD, and 6 are reported to manifest autistic behaviors. This is a marked contrast to the $27 \%$ of patients with PWS who are diagnosed with ASD. ${ }^{13}$ Notable cognitive behavioral characteristics were described as being impulsive, compulsive, varying degrees of stubbornness, and being manipulative. Detailed neuropsychological phenotyping would be warranted to further delineate the behavioral phenotypes of individuals with MAGEL2 loss of function. Of note, a Magel2-deficient mouse model manifests deficits in social recognition and social interaction. ${ }^{14}$

It is readily apparent that patients with truncating mutations of MAGEL2 express different levels of severity. On one hand, multiple patients have relatively mild contractures, affecting only the interphalangeal joint. On the other hand, some patients with fetal akinesia have severely decreased fetal movement and arthrogryposis multiplex, as seen in patient 5 and 6 (Supplementary Table S1 online; Supplementary Figure S1 online). Indeed, several individuals who appeared to be affected with SHFYNG died during childhood. Reported causes of death included apnea, aspiration pneumonia with subsequent medical complications, and sudden infant death syndrome. However, further investigation of the natural course of SHFYNG and its associated mortality is necessary.

Nucleotides c.1990-1996 represent a sequence of seven cytosines, which seems to represent a mutational hotspot, with a molecularly confirmed diagnosis in a total of 13 individuals from 8 families with a c.1996dupC pathogenic variant and 5 individuals from 2 families with a c.1996delC pathogenic variant. The fact that the two cases of fetal akinesia reported herein carried the same pathogenic variant (c.1996delC; p.Q666fs) as the three siblings with prenatally lethal arthrogryposis multiplex reported by Mejlachowicz et al. ${ }^{4}$ suggests that some genotype-phenotype correlations may exist. In this context it is worth noting that neither missense mutations in MAGEL2 nor mutations of the MAGEL2 promoter have been reported to date. These may provide further information regarding genotype-phenotype correlation.

While we are able to present the clinical phenotypes of 18 patients with a molecularly confirmed diagnosis, the ability to gain a complete medical history and background can prove troublesome. Because of a family's geographic location, the lack of historical medical information, and the availability of care, not all phenotypes can be assessed. Moreover, the age of an individual can predict the availability of phenotypic information, such as a diagnosis of ASD, characteristic cognitive behaviors, and hypogonadism in males. While male hypogonadism can often be noted clinically, even at the time of birth, via the observation of cryptorchidism and/or micropenis, female hypogonadism is not as readily recognizable. As mentioned, patient 18 is the oldest female in our cohort and the only female reported with hypogonadism to date. She was diagnosed with hypogonadotropic hypogonadism and presented dysmorphic labial development that was identifiable upon pubertal maturation. A systematic, endocrinological assessment of individuals with SHFYNG has not been reported to date.

Given the phenotypic overlap between PWS and SHFYNG, testing for mutations in MAGEL2 should be considered in the context of PWS-like phenotypes but negative PWS methylation analysis. In fact, during infancy, PWS was considered high on the differential diagnosis for most of the individuals reported herein. While SHFYNG and PWS share an appreciable number of common features, and no specific feature seems to be exclusive of one or the other condition, the presence of contractures at birth makes SHFYNG more likely, whereas the development of hyperphagia during childhood seems to be more characteristic of PWS. The overlap and the distinction between the two syndromes has been described previously. ${ }^{15}$

PWS and a PWS-like phenotype usually represent sporadic disorders, with single individuals affected within a given family. This report of 18 individuals includes three families carrying a truncating MAGEL2 mutation leading to SHFYNG. The familial association highlights the importance of parental testing, determination of the allelic location of the mutation, and the challenges for genetic counseling. Unlike "regular" autosomal 
dominant disorders, the imprinted nature of this condition can phenotypically skip several generations, as long as the mutation resides on the maternal (imprinted) chromosome. However, the chance that the offspring of male individuals carrying a deleterious MAGEL2 mutation to be clinically affected is $50 \%$.

For several individuals and their families reported herein, making a molecular diagnosis puts an end to a long diagnostic odyssey. It allows families to connect with each other to exchange experience and knowledge and to receive emotional support. In particular, these families have connected via social media, providing support to families with newly identified affected individuals, showing the important role of family advocacy, especially in rare disorders such as SHFYNG.

Current trials are under way to evaluate oxytocin as a potential therapy for PWS (ClinicalTrials.gov identifiers NCT01944046 and NCT02629991). Oxytocin therapy has been shown to be effective in Magel2-null mice, and it may be beneficial for humans expressing truncating mutations of MAGEL2 as well. ${ }^{14,16}$ Having families connected together will allow for a quick dissemination of updated knowledge and faster enrollment into potential clinical trials.

\section{SUPPLEMENTARY MATERIAL}

Supplementary material is linked to the online version of the paper at http://www.nature.com/gim

\section{ACKNOWLEDGMENTS}

This work was supported by the Joan and Stanford Alexander Family (C.P.S.), and the Foundation for Prader-Willi Research (C.P.S.). This research is supported by the Intellectual and Developmental Disabilities Research Center (1U54 HD083092). The authors are indebted to the patients and their families for their willingness to participate in this study.

A Facebook group for affected families is at http://www.facebook.com/MAGEL2.

\section{DISCLOSURE}

The authors declare no conflict of interest.

\section{REFERENCES}

1. Schaaf CP, Gonzalez-Garay ML, Xia F, et al. Truncating mutations of MAGEL2 cause Prader-Willi phenotypes and autism. Nat Genet 2013;45:1405-1408.

2. Holm VA, Cassidy SB, Butler MG, et al. Prader-Willi syndrome: consensus diagnostic criteria. Pediatrics 1993;91:398-402.

3. Soden SE, Saunders CJ, Willig LK, et al. Effectiveness of exome and genome sequencing guided by acuity of illness for diagnosis of neurodevelopmental disorders. Sci Trans/ Med 2014;6:265ra168.

4. Mejlachowicz D, Nolent F, Maluenda J, et al. Truncating mutations of MAGEL2, a gene within the Prader-Willi locus, are responsible for severe arthrogryposis. Am J Hum Genet 2015;97:616-620.

5. Kanber D, Giltay J, Wieczorek D, et al. A paternal deletion of MKRN3, MAGEL2 and NDN does not result in Prader-Willi syndrome. Eur J Hum Genet 2009; 17:582-590.

6. Buiting K, Di Donato N, Beygo J, et al. Clinical phenotypes of MAGEL2 mutations and deletions. Orphanet J Rare Dis 2014;9:40.

7. Matarazzo V, Muscatelli F. Natural breaking of the maternal silence at the mouse and human imprinted Prader-Willi locus: a whisper with functional consequences. Rare Dis 2013;1:e27228.

8. Denizot S, Boscher C, Le Vaillant C, Rozé JC, Gras Le Guen C. Distal arthrogryposis and neonatal hypotonia: an unusual presentation of Prader-Willi syndrome (PWS). J Perinatol 2004;24:733-734.

9. Bigi N, Faure JM, Coubes C, et al. Prader-Willi syndrome: is there a recognizable fetal phenotype? Prenat Diagn 2008;28:796-799.

10. Haugen G, Rønnestad A, Kroken M. Variations in fetal phenotype in Prader-Willi syndrome. Prenat Diagn 2009;29:294.

11. Miller JL, Lynn CH, Driscoll DC, et al. Nutritional phases in Prader-Willi syndrome. Am J Med Genet A 2011;155A:1040-1049.

12. Bischof JM, Stewart CL, Wevrick R. Inactivation of the mouse Magel2 gene results in growth abnormalities similar to Prader-Willi syndrome. Hum Mol Genet 2007;16:2713-2719.

13. Bennett JA, Germani T, Haqq AM, Zwaigenbaum L. Autism spectrum disorder in Prader-Willi syndrome: A systematic review. Am J Med Genet A 2015;167:2936-2944.

14. Meziane $H$, Schaller $F$, Bauer $S$, et al. An early postnatal oxytocin treatment prevents social and learning deficits in adult mice deficient for Magel2, a gene involved in Prader-Willi syndrome and autism. Biol Psychiatry 2015;78:85-94.

15. Fountain MD, Schaaf CP. Prader-Willi syndrome and Schaaf-Yang syndrome: neurodevelopmental diseases intersecting at the MAGEL2 gene. Diseases 2016;4. http://www.mdpi.com/2079-9721/4/1/2

16. Fountain MD Jr, Schaaf CP. MAGEL2 and Oxytocin-Implications in Prader-Willi Syndrome and Beyond. Biol Psychiatry 2015;78:78-80. 